\title{
A Note on the Stability Analysis of Fuzzy Nonlinear Fractional Differential Equations Involving the Caputo Fractional Derivative
}

\author{
Ali El Mfadel $(\mathbb{D}$, Said Melliani $\mathbb{D}$, and M'hamed Elomari \\ Laboratory of Applied Mathematics and Scientific Computing, Sultan Moulay Slimane University, Beni Mellal, Morocco \\ Correspondence should be addressed to Ali El Mfadel; elmfadelali@gmail.com
}

Received 14 May 2021; Revised 19 July 2021; Accepted 1 September 2021; Published 13 September 2021

Academic Editor: Remi Léandre

Copyright (C) 2021 Ali El Mfadel et al. This is an open access article distributed under the Creative Commons Attribution License, which permits unrestricted use, distribution, and reproduction in any medium, provided the original work is properly cited.

In this paper, we present and establish a new result on the stability analysis of solutions for fuzzy nonlinear fractional differential equations by extending Lyapunov's direct method from the fuzzy ordinary case to the fuzzy fractional case. As an application, several examples are presented to illustrate the proposed stability result.

\section{Introduction}

The theory of fuzzy fractional differential equations was initiated by Agarwal et al. in [1] who proposed the concept of solutions for fractional differential equations with uncertainties, and they considered the Riemann-Liouville differentiability to solve the equations which is a combination of the Hukuhara difference and Riemann-Liouville derivative. Arshad and Lupulescu in $[2,3]$ developed the theory of the fractional calculus for interval-valued functions and proved some results on the existence and uniqueness of the solution for the fuzzy fractional differential equations. Later, Alikhani and Bahrami in [4] proved the existence and uniqueness results for nonlinear fuzzy fractional integral and integrodifferential equations by using the method of upper and lower solutions. The authors in [5-7] discussed the concepts about the generalized Hukuhara fractional Riemann-Liouville and Caputo differentiability of fuzzy-valued functions, and the equivalence between the fuzzy fractional differential equation and fuzzy fractional integral equation was discussed in [8]. On the contrary, numerical solutions of interval-valued fractional nonlinear differential equations were investigated in $[9,10]$.

Recently, fractional calculus was introduced to the stability analysis of nonlinear systems. The stability notion is one of the most important issues for differential equations, although this problem is over many years. Stability of fractional differential systems has attracted increasing interest. For example, the earliest study on the stability of fractional differential equations started in [11]. For more details about the stability results and the methods available to analyze the stability of fractional differential equations, the reader may refer to the recent papers [12-14] and the references therein.

In this paper, our aim is to study the stability result of the following fuzzy nonlinear fractional differential equation:

$$
\left\{\begin{array}{l}
{ }^{c} D_{0^{+}}^{q} u(t)=f(t, u(t)) ; \quad t \in J=[0,+\infty[ \\
u(0)=u_{0},
\end{array}\right.
$$

by extending Lyapunov's direct method from the ordinary fuzzy case to the fractional fuzzy case such that $f: J \times E^{1} \longrightarrow E^{1}$ is a fuzzy continuous function in $t$ and locally Lipschitz in $u$ and ${ }^{c} D_{0^{+}}^{q}$ is the Caputo fractional derivative of $u(t)$ at order $0<q<1$.

Our paper is organized as follows: Section 2 gives some basic definitions, lemmas, and theorems as preliminaries of the fuzzy set theory and fuzzy fractional calculus. In Section 3 , we give some sufficient criteria to guarantee the stability of the trivial solution for fuzzy nonlinear fractional differential equation (1), and we present an example to illustrate the proposed stability result. Finally, in Section 4, this work is concluded by conclusion and future works. 


\section{Preliminaries}

Here, we review some essential facts from fuzzy fractional calculus, basic definitions of a fuzzy number, and fuzzy concepts.

Definition 1 (see [15]). A fuzzy number is mapping $u: \mathbb{R} \longrightarrow[0,1]$ such that

(1) $u$ is upper semicontinuous

(2) $u$ is normal; that is, there exists $x_{0} \in \mathbb{R}$ such that $u\left(x_{0}\right)=1$

(3) $u$ is fuzzy convex; that is, $u(\lambda x+(1-\lambda) y)$ $\geq \min \{u(x), u(y)\}$ for all $x, y \in \mathbb{R}$ and $\lambda \in[0,1]$

(4) $\overline{\{x \in \mathbb{R}, u(x)>0\}}$ is compact

The $\alpha$ - cut of a fuzzy number $u$ is defined as follows:

$$
[u]^{\alpha}=\{x \in \mathbb{R} \backslash u(x) \geq \alpha\} .
$$

Moreover, we can also present the $\alpha$-cut of fuzzy number $u$ by $[u]^{\alpha}=\left[u_{l}(\alpha), u_{r}(\alpha)\right]$.

Notations:

We denote by $E^{1}$ the collection of all fuzzy numbers. We also denote by $0_{E^{1}}$ the fuzzy zero defined by

$$
0_{E^{1}}(x)= \begin{cases}1, & \text { if } x=0, \\ 0, & \text { elswhere }\end{cases}
$$

Example 1. Let $u$ be a fuzzy number defined by the following function:

$$
\mu_{u}(x)= \begin{cases}x-1, & x \in[1,2], \\ -x+3, & x \in[2,3], \\ 0, & \text { elswhere. }\end{cases}
$$

Then, we have $[u]^{1}=\{2\}$.

Definition 2 (see [8]). Let $u \in E^{1}$ and $\alpha \in[0,1]$; we define the diameter of the $\alpha$ - level set of the fuzzy set $u$ as follows:

$$
d\left([u]^{\alpha}\right)=u_{r}-u_{l} .
$$

We denote by $C\left(J, E^{1}\right)$ the space of all fuzzy-valued functions which are continuous on $J$ and $\mathbb{P}_{c}(\mathbb{R})$ the space of all the compact subsets of $\mathbb{R}$.

Definition 3 (see [8]). The generalized Hukuhara difference of two fuzzy numbers $u, v \in E^{1}$ is defined as follows:

$$
u \ominus_{g H} v=w \Longleftrightarrow\left\{\begin{array}{l}
\text { i) } u=v+w, \\
\text { or } \\
\text { ii) } v=u+(-1) w .
\end{array}\right.
$$

Property 1 (see [8]). If $u \in E^{1}$ and $v \in E^{1}$, then the following properties hold:
(1) If $u \ominus_{g H} v$ exists, then it is unique

(2) $u \ominus_{g H} u=0_{E^{1}}$

(3) $(u+v) \ominus_{g H} v=u$

(4) $u \ominus_{g H} v=0_{E^{1}} \Longleftrightarrow u=v$

Definition 4 (see [15]). According to Zadeh's extension principle, the addition on $E^{1}$ is defined by

$$
(u \oplus v)(z)=\sup _{z=x+y} \min \{u(x), v(y)\} .
$$

And scalar multiplication of a fuzzy number is given by

$$
(k \odot u)(x)= \begin{cases}u\left(\frac{x}{k}\right), & k>0, \\ 0_{E^{1}}, & k=0 .\end{cases}
$$

Remark 1 (see [16]). Let $u, v \in E^{1}$ and $\alpha \in[0,1]$; then, we have

$$
\begin{aligned}
{[u+v]^{\alpha} } & =[u]^{\alpha}+[v]^{\alpha}, \\
{[u-v]^{\alpha} } & =\left[u_{1}^{\alpha}-v_{2}^{\alpha}, u_{2}^{\alpha}-v_{1}^{\alpha}\right], \\
{[k u]^{\alpha} } & =k[u]^{\alpha}= \begin{cases}{\left[\lambda u_{1}^{\alpha}, \lambda u_{2}^{\alpha}\right],} & \text { if } \lambda \geq 0, \\
{\left[\lambda u_{2}^{\alpha}, \lambda u_{1}^{\alpha}\right],} & \text { if } \lambda<0,\end{cases} \\
{[u v]^{\alpha} } & =\left[\min u_{1}^{\alpha} v_{1}^{\alpha}, u_{1}^{\alpha} v_{2}^{\alpha}, u_{2}^{\alpha} v_{1}^{\alpha}, u_{2}^{\alpha} v_{2}^{\alpha}, \max u_{1}^{\alpha} v_{1}^{\alpha}, u_{1}^{\alpha} v_{2}^{\alpha}, u_{2}^{\alpha} v_{1}^{\alpha}, u_{2}^{\alpha} v_{2}^{\alpha}\right] .
\end{aligned}
$$

Definition 5 (see [16]). Let $u, v \in E^{1}$ with $\alpha \in[0,1]$; then, the Hausdorff distance between $u$ and $v$ is given by

$$
D(u, v)=\sup _{\alpha \in[0,1]} d\left([u]^{\alpha},[v]^{\alpha}\right),
$$

where $d$ is the Hausdorff metric defined in $P_{c}(R)$.

Proposition 1 (see [17]). D is a metric on $E^{1}$ and has the following properties:

(1) $\left(E^{1} ; D\right)$ is a complete metric space

(2) $D(u+w, v+w)=D(u, v)$

(3) $D(k u, k v)=|k| D(u, v), \forall u, v \in E^{1}$ and $k \in R$

(4) $D(u+w, v+z) \leq D(u, v)+D(w, z), \forall u, v, w, z \in E^{1}$

Definition 6 (see [18]). Let $f: J \longrightarrow E^{1}$ and $t_{0} \in J$. We say that $f$ is Hukuhara differentiable at $t_{0}$ if there exists $f^{\prime}\left(t_{0}\right) \in E^{1}$ such that

$$
\begin{aligned}
f^{\prime}\left(t_{0}\right) & =\lim _{h \longrightarrow 0^{+}} \frac{f\left(t_{0}+h\right) \ominus_{g H} f\left(t_{0}\right)}{h} \\
& =\lim _{h \longrightarrow 0^{-}} \frac{f\left(t_{0}\right) \ominus_{g H} f\left(t_{0}-h\right)}{h} .
\end{aligned}
$$

Remark 2. Let $f: J \longrightarrow E^{1}$ be a fuzzy function such that $[f(x)]^{\alpha}=[\underline{f}(x ; \alpha), \bar{f}(x ; \alpha)]$ for each $\alpha \in[0,1]$; then, 


$$
\left[f^{\prime}(x)\right]^{\alpha}=\left[\underline{f^{\prime}}(x ; \alpha), \overline{f^{\prime}}(x ; \alpha)\right] .
$$

Definition 7 (see [17]). $F: J \longrightarrow E^{1}$ is strongly measurable if $\forall \alpha \in[0,1]$, the set-valued mapping $F_{\alpha}: J \longrightarrow \mathrm{P}_{c}(\mathbf{R})$ defined by $F_{\alpha}(t)=[F(t)]^{\alpha}$ is Lebesgue measurable.

A function $F: J \longrightarrow E^{1}$ is called integrably bounded if there exists an integrable function $h$ such that $|x|<h(t)$, $\forall x \in F_{0}(t)$.

Definition 8 (see [19]). Let $F: J \longrightarrow E^{1}$. The integral of $F$ on $J$ denoted by $\int_{J} F(t) \mathrm{d} t$ is given by

$$
\begin{aligned}
{\left[\int_{J} F(t) \mathrm{d} t\right]^{\alpha} } & =\int_{J} F_{\alpha}(t) \mathrm{d} t \\
& =\left\{\int_{J} f(t) \mathrm{d} t \mid f: J \longrightarrow \mathbb{R} \text { is a measurable selection for } F_{\alpha}\right\},
\end{aligned}
$$

for all $\alpha \in[0,1]$.

Proposition 2 (see [17]). Let $F: J \longrightarrow E^{1}$ be a fuzzy function. If $F$ is strongly measurable and integrably bounded, then it is integrable.

\subsection{Fractional Integral and Fractional Derivative of the Fuzzy Function}

Proposition 3 (see [17]). If $u \in E^{1}$, then the following properties hold:

(1) $[u]^{\beta} \subset[u]^{\alpha}$ if $0 \leq \alpha \leq \beta$.

(2) If $\alpha_{n} \subset[0,1]$ is a nondecreasing sequence which converges to $\alpha$, then

$$
[u]^{\alpha}=\bigcap_{n \geq 1}[u]^{\alpha_{n}} .
$$

Conversely, if $A^{\alpha}=\left\{\left[u_{1}^{\alpha}, u_{2}^{\alpha}\right] ; \alpha \in[0,1]\right\}$ is a family of closed real intervals verifying (1) and (16), then $A^{\alpha}$ defined a fuzzy number $u \in E^{1}$ such that $[u]^{\alpha}=A^{\alpha}$.

Let $0<q<1$; the fractional integral of order $q$ of a real function $g: J \longrightarrow R$ is given by

$$
I_{0^{+}}^{q} g(t)=\frac{1}{\Gamma(q)} \int_{0}^{t}(t-s)^{q-1} g(s) \mathrm{d} s
$$

Let $f(t) \in L\left(J, E^{1}\right)$ such that $f(t)=\left[f_{1}^{\alpha}(t), f_{2}^{\alpha}(t)\right]$. Suppose that $f_{1}^{\alpha}, f_{2}^{\alpha} \in L(J, \mathbb{R})$ for all $\alpha \in[0,1]$, and let

$$
A^{\alpha}=\left[\frac{1}{\Gamma(q)} \int_{0}^{t}(t-s)^{q-1} f_{1}^{\alpha}(s) \mathrm{d} s, \frac{1}{\Gamma(q)} \int_{0}^{t}(t-s)^{q-1} f_{2}^{\alpha}(s) \mathrm{d} s\right],
$$

where $\Gamma(\cdot)$ is the Euler gamma function.

We have the following lemma.
Lemma 1 (see [1]). The family $\left\{A^{\alpha} ; \alpha \in[0,1]\right\}$ given by (16) defined a fuzzy number $u \in E^{1}$ such that $[u]^{\alpha}=A^{\alpha}$.

Definition 9 (see [6]). Let $f(t) \in L\left(J, E^{1}\right)$.

The fuzzy fractional integral of order $q \in[0,1]$ of $f$ denoted by

$$
I_{0^{+}}^{q} f(t)=\frac{1}{\Gamma(q)} \int_{0}^{t}(t-s)^{q-1} f(s) \mathrm{d} s
$$

is defined by

$$
\left[I_{0^{+}}^{q} f(t)\right]^{\alpha}=\left[I_{0^{+}}^{q} f_{l}(t ; \alpha), I_{0^{+}}^{q} f_{r}(t ; \alpha)\right] .
$$

Proposition 4 (see [6]). Let $f, g \in L\left(J, E^{1}\right), q \in[0,1]$, and $b \in E^{1}$; then, we have

(1) $I_{0^{+}}^{q}(b f)(t)=b I_{0^{+}}^{q} f(t)$

(2) $I_{0^{+}}^{q}(f+g)(t)=I_{0^{+}}^{q} f(t)+I_{0^{+}}^{q} g(t)$

(3) $I_{0^{+}}^{q_{1}} I_{0^{+}}^{q_{2}} f(t)=I_{0^{+}}^{q_{1}+q_{2}} f(t)$, where $\left(q_{1}, q_{2}\right) \in[0,1]^{2}$

Example 2. Let $u(t)=u \in E^{1}$ such that $[u]^{\alpha}=\left[u_{\alpha}^{1}, u_{\alpha}^{2}\right]$; then,

$$
\begin{aligned}
{\left[I_{0^{+}}^{q} u(t)\right]^{\alpha}=} & {\left[\frac{1}{\Gamma(q)} \int_{0}^{t}(t-s)^{q-1} u_{\alpha}^{1}(s) \mathrm{d} s,\right.} \\
& \left.\frac{1}{\Gamma(q)} \int_{0}^{t}(t-s)^{q-1} u_{\alpha}^{2}(s) \mathrm{d} s\right], \\
{\left[I_{0^{+}}^{q} u(t)\right]^{\alpha}=} & \frac{t^{q}}{\Gamma(q+1)}\left[u_{\alpha}^{1}, u_{\alpha}^{2}\right], \\
{\left[I_{0^{+}}^{q} u(t)\right]^{\alpha}=} & \frac{t^{q}}{\Gamma(q+1)}[u]^{\alpha} .
\end{aligned}
$$

Definition 10 (see [6]). Let $f \in C\left(J, E^{1}\right) \cap L\left(J, E^{1}\right)$. The function $f$ is called fuzzy Caputo fractional differentiable of order $0<q<1$ at $t$ if there exists an element ${ }^{c} D_{0^{+}}^{q} f(t) \in E^{1}$ such that

$$
{ }^{c} D_{0^{+}}^{q} f(t)=\frac{1}{\Gamma(q)} \int_{0}^{t}(t-s)^{q-1} f^{\prime}(s) \mathrm{d} s .
$$

Remark 3 (see [6]). Since $[f(t)]^{\alpha}=\left[f_{l}(t ; \alpha), f_{r}(t ; \alpha)\right]$ for each $\alpha \in[0,1]$,

$$
\left[{ }^{c} D_{0^{+}}^{q} f(t)\right]^{\alpha}=\left[{ }^{c} D_{0^{+}}^{q} f_{l}(t ; \alpha),{ }^{c} D^{q} f_{r}(t ; \alpha)\right],
$$

where

$$
\begin{aligned}
& { }^{c} D_{0^{+}}^{q} f_{l}(t ; \alpha):=\frac{1}{\Gamma(q)} \int_{0}^{t}(t-s)^{q-1} f_{l}^{\prime}(s, \alpha) \mathrm{d} s, \\
& { }^{c} D_{0^{+}}^{q} f_{r}(t ; \alpha):=\frac{1}{\Gamma(q)} \int_{0}^{t}(t-s)^{q-1} f_{r}^{\prime}(s, \alpha) \mathrm{d} s .
\end{aligned}
$$

Example 3. Let $u(t)=u \in E^{1}$. If $[u]^{\alpha}=\left[u_{\alpha}^{1}, u_{\alpha}^{2}\right]$, then 


$$
\begin{aligned}
{\left[{ }^{c} D_{0^{+}}^{q} u(t)\right]^{\alpha}=} & {\left[\frac{1}{\Gamma(q)} \int_{0}^{t}(t-s)^{q-1}\left(u_{\alpha}^{1}\right)^{\prime} \mathrm{d} s,\right.} \\
& \left.\frac{1}{\Gamma(q)} \int_{0}^{t}(t-s)^{q-1}\left(u_{\alpha}^{2}\right)^{\prime} \mathrm{d} s\right], \\
{\left[{ }^{c} D_{0^{+}}^{q} u(t)\right]^{\alpha}=} & \{0\}, \\
{ }^{c} D_{0^{+}}^{q} u(t)= & 0_{E^{1}} .
\end{aligned}
$$

2.2. Laplace Transform of the Caputo Fractional Derivative. For establishing the Laplace transform of the Caputo fractional derivative [20], we write the Caputo derivative under the form

$$
{ }^{c} D_{0^{+}}^{\beta} f(t):=I^{n-\beta} f^{(n)}(t),
$$

where $\beta>0$ such that $n<\beta<n+1$.

By using the formula of the Laplace transform of the Riemann-Liouville fractional integral, we have

$$
L\left\{{ }^{c} D^{\alpha} f(t), s\right\}:=L\left\{I^{n-\beta} f^{(n)}(t), s\right\}=s^{\beta-n} G(s),
$$

where $G(s)$ is given by

$$
G(s)=s^{n} F(s)-\sum_{k=0}^{n-1} s^{n-k-1} f^{(k)}(0)=s^{n} F(s)-\sum_{k=0}^{n-1} s^{k} f^{(n-k-1)}(0) .
$$

Finally, the Laplace transform of the Caputo fractional derivative is given by

$$
L\left\{{ }^{c} D^{\beta} f(t), s\right\}=s^{\beta} F(s)-\sum_{k=0}^{n-1} s^{\beta-k-1} f^{(k)}(0) .
$$

Remark 4 (see [20]). If $0<\beta<1$, then we have

$$
L\left\{{ }^{c} D^{\beta} f(t), s\right\}=s^{\beta} F(s)-f(0) .
$$

2.3. Laplace Transform of the Mittag-Leffler Function. The Mittag-Leffler function is an important function that finds widespread use in the world of fractional calculus. Just as the exponential naturally arises out of the solution to integerorder differential equations, the Mittag-Leffler function plays an analogous role in the solution of noninteger-order differential equations.

Definition 11 (see [20]). We recall that the Mittag-Leffler function is given by

$$
E_{\alpha}(z)=\sum_{k=0}^{+\infty} \frac{z^{k}}{\Gamma(\alpha k+1)}, \quad \alpha \in \mathbb{C}, R(\alpha)>0, z \in \mathbb{C} .
$$

And the general form is given by

$$
E_{\alpha, \beta}(z)=\sum_{k=0}^{+\infty} \frac{z^{k}}{\Gamma(\alpha k+\beta)}, \quad \beta, \alpha \in \mathbb{C}, R(\beta)>0, R(\alpha)>0, z \in \mathbb{C} .
$$

Then, we have

$$
L\left\{E_{\alpha}\left(\lambda t^{\alpha}\right) ; s\right\}=\frac{s^{\alpha-1}}{s^{\alpha}-\lambda}, \quad s>\lambda^{1 / \alpha} .
$$

Indeed, for $s>\lambda$, using the series expansion of the exponential function, we have

$$
\begin{aligned}
\frac{1}{s-\lambda} & =\in 0^{+\infty} e^{-s t} e^{\lambda t} \mathrm{~d} t \\
& =\sum_{k=0}^{\infty} \frac{\lambda^{k}}{k !} \in 0^{+\infty} e^{-s t} t^{k} \mathrm{~d} t \\
& =\sum_{k=0}^{\infty} \frac{\lambda^{k}}{k !} \frac{k !}{s^{k+1}} \\
& =\sum_{k=0}^{\infty} \frac{\lambda^{k}}{s^{k+1}} .
\end{aligned}
$$

Then, similarly, for the Mittag-Leffler function, we obtain

$$
\begin{aligned}
& L\left\{E_{\alpha}\left(\lambda t^{\alpha}\right) ; s\right\}=\sum_{k=0}^{\infty} \frac{\lambda^{k}}{\Gamma(\alpha k+1)} \in 0^{+\infty} e^{-s t} t^{\alpha k} \mathrm{~d} t, \\
& L\left\{E_{\alpha}\left(\lambda t^{\alpha}\right) ; s\right\}=\sum_{k=0}^{\infty} \frac{\lambda^{k}}{\Gamma(\alpha k+1)} \frac{\Gamma(\alpha k+1)}{s^{\alpha k+1}}, \\
& L\left\{E_{\alpha}\left(\lambda t^{\alpha}\right) ; s\right\}=\sum_{k=0}^{\infty} \frac{\lambda^{k}}{s^{\alpha k+1}}=s^{\alpha-1} \sum_{k=0}^{\infty} \frac{\lambda^{k}}{\left(s^{\alpha}\right)^{k+1}}, \\
& L\left\{E_{\alpha}\left(\lambda t^{\alpha}\right) ; s\right\}==\frac{s^{\alpha-1}}{s^{\alpha}-\lambda}, \quad s>\lambda^{1 / \alpha} .
\end{aligned}
$$

The Laplace transform of the Mittag-Leffler function in two parameters $(\alpha, \beta) \in \mathbb{C}$ is given by

$$
L\left\{t^{\beta-1} E_{\alpha, \beta}\left(-\lambda t^{\alpha}\right) ; s\right\}=\frac{s^{\alpha}-\beta}{s^{\alpha}+\lambda}, \quad R(s)>|\lambda|^{1 / \alpha} .
$$

\section{Main Result}

The existence and uniqueness results of the solution for problem (1) are discussed in [21] by using the continuity and Lipschitz conditions of the function $f$.

In this paper, we suppose that $f\left(t, 0_{E^{1}}\right)=0_{E^{1}}$; this fact means that the fuzzy zero function is a solution of problem (1). We extend the Lyapunov direct method of stability to introduce Mittag-Leffler stability analysis of the fuzzy trivial solution $\left(u=0_{E^{1}}\right)$ for problem (1).

Definition 12. A fuzzy number $u_{e}$ is an equilibrium point of system (1) if and only if $f\left(t, u_{e}\right)=0_{E^{1}}$. 
Remark 5. For convenience, we state all definitions and theorems for the case when the equilibrium point is the origin $u_{e}=0_{E^{1}}$.

There is no loss of generality in doing so because any equilibrium point can be shifted to the origin via a change of variables.

Suppose that the fuzzy equilibrium point for (1) is $u_{e} \neq 0_{E^{1}}$, and consider the change of variable $U=u \ominus_{g H} u_{e}$. The fractional derivative of $U(t)$ is given by

$$
{ }^{c} D_{0^{+}}^{q} U(t)={ }^{c} D_{0^{+}}^{q}\left(u(t) \ominus_{g H} u_{e}\right)=f\left(t, u(t)+u_{e}\right)=F(t, u(t)),
$$

where $F\left(t, 0_{E^{1}}\right)=0_{E^{1}}$ and the system has equilibrium at the origin.

Definition 13. The trivial solution $0_{E^{1}}$ of problem (1) is said to be

(1) Stable iff for any $\epsilon>0$, there exists $\delta>0$ such that

$$
D\left(u_{0}, 0_{E^{1}}\right)<\delta \Rightarrow D\left(u(t), 0_{E^{1}}\right)<\epsilon, \quad \forall t \geq 0 .
$$

(2) Asymptotically stable iff $\lim _{t \longrightarrow+\infty} D\left(u(t), 0_{E^{1}}\right)=0$.

Definition 14. The solution of (1) is said to be Mittag-Leffler stable if

$$
D\left(u(t), 0_{E^{1}}\right) \leq\left\{m\left(u_{0}\right) E_{q}\left(-\lambda t^{q}\right)\right\}^{b},
$$

where $0<q<1, \lambda \geq 0, b>0, m(0)=0$, and $m(u) \geq 0$ and $m(u)$ is locally Lipschitz on $u$ with Lipschitz constant $m_{0}$.

Remark 6. The Mittag-Leffler stability implies the stability of Lyapunov.

Theorem 1. Let $V(t, u(t)):\left[0,+\infty\left[\times E^{1} \longrightarrow \mathbb{R}\right.\right.$ be a continuously differentiable function and locally Lipschitz with respect to $u$ such that

$$
\begin{gathered}
{ }_{c_{1}} D^{a}\left(u(t), 0_{E^{1}}\right) \leq V(t, u(t)) \leq_{c_{2}} D^{a b}\left(u(t), 0_{E^{1}}\right), \\
{ }^{c} D_{0^{+}}^{\beta}(V(t, u(t))) \leq{ }_{-c_{3}} D^{a b}\left(u(t), 0_{E^{1}}\right),
\end{gathered}
$$

where $t \geq 0, \beta \in[0,1]$, and $c_{1}, c_{2}, c_{3}, a$, and $b$ are positive constants; then, the trivial solution of (1) is Mittag-Leffler stable.

Proof. From equations (38) and (39), we obtain

$$
{ }^{c} D_{0^{+}}^{\beta}(V(t, u(t))) \leq \frac{-c_{3}}{c_{2}} V(t, u(t)) .
$$

There exists a positive function $k(t)$ satisfying

$$
{ }^{c} D_{0^{+}}^{\beta}(V(t, u(t)))+k(t)=\frac{-c_{3}}{c_{2}} V(t, u(t)) .
$$

By using the Laplace transform (27), we get

$$
\begin{aligned}
& S^{\beta} L\{V(t, u(t)) ; s\}-S^{\beta-1} V(0, u(0))+L\{k(t) ; s\} \\
& =\frac{-c_{3}}{c_{2}} L\{V(t, u(t)) ; s\} .
\end{aligned}
$$

It follows that

$$
L\{V(t, u(t)) ; s\}=\frac{S^{\beta-1} V(0, u(0))-L\{k(t) ; s\}}{S^{\beta}+\left(c_{3} / c_{2}\right)} .
$$

By using the inverse of the Laplace transform, we obtain $V(t, u(t))=E_{\beta}\left(\frac{-c_{3}}{c_{2}} t^{\beta}\right) V(0, u(0))-k(t) *\left(t^{1-\beta} E_{\beta, \beta}\left(\frac{-c_{3}}{c_{2}} t^{\beta}\right)\right)$.

Since $E_{\beta, \beta}\left(\left(-c_{3} / c_{2}\right) t^{\beta}\right)$ is a nonnegative function (see [20]), it follows that

$$
\begin{gathered}
V(t, u(t)) \leq E_{\beta}\left(\frac{-c_{3}}{c_{2}} t^{\beta}\right) V(0, u(0)), \\
D\left(u(t), 0_{E^{1}}\right) \leq\left[\frac{V(0)}{c_{1}} E_{\beta}\left(\frac{-c_{3}}{c_{2}} t^{\beta}\right)\right]^{1 / a},
\end{gathered}
$$

where $V(0)=V(0, u(0))$.

Let $m=\left(V(0) / c_{1}\right)$; then, we have

$$
D\left(u(t), 0_{E^{1}}\right) \leq\left[m E_{\beta}\left(\frac{-c_{3}}{c_{2}} t^{\beta}\right)\right]^{1 / a}
$$

where $m=0$ if and only if $u(0)=0_{E^{1}}$.

Since $V(t, u)$ is locally Lipschitz with respect to $x$, it follows that $m=\left(V(0, u(0)) / c_{1}\right)$ is Lipschitz with respect to $u(0)$ and $m(0)=0$ which imply the Mittag-Leffler stability of system (1).

3.1. Illustrative Example. The following example is used to demonstrate the applicability of our stability result. We consider the following fuzzy nonlinear system:

$$
\left\{\begin{array}{l}
{ }^{c} D_{0^{+}}^{q} u(t)=f(t, u(t)) \\
u(0)=u_{0}
\end{array}\right.
$$

where $0<q<1, u_{e}=0_{E^{1}}$ is the equilibrium point of problem (47), and $f: J \times E^{1} \longrightarrow E^{1}$ is a fuzzy Lipschitz function with Lipschitz constant $k>0$.

We suppose that there exists a Lyapunov function $V(t, u(t))$ satisfying the following conditions:

$$
\begin{gathered}
{ }_{c_{1}} D\left(u(t), 0_{E^{1}}\right) \leq V(t, u(t)) \leq_{c_{2}} D\left(u(t), 0_{E^{1}}\right), \\
V^{\prime}(t, u) \leq{ }_{-_{3}} D\left(u, 0_{E^{1}}\right),
\end{gathered}
$$

where $c_{1}, c_{2}$, and $c_{3}$ are positive constants and $V^{\prime}(t, u)=$ $(\mathrm{d} V(t, u(t)) / \mathrm{d} t)$.

By using inequalities (48) and (49), we can write 


$$
{ }^{c} D_{0^{+}}^{1-q}\left(V(t, u(t))=I_{0^{+}}^{q} V^{\prime}(t, u) \leq_{-c_{3}} I_{0^{+}}^{q} D\left(u(t), 0_{E^{1}}\right) .\right.
$$

Since the fuzzy function $f: J \times E^{1} \longrightarrow E^{1}$ is Lipschitz with a real constant $k>0$, it follows that

$$
D\left(f(t, u), 0_{E^{1}}\right) \leq k, \quad D\left(u(t), 0_{E^{1}}\right) .
$$

Thus,

$$
\begin{aligned}
& { }^{c} D_{0^{+}}^{1-q}(V(t, u(t))) \leq \frac{-c_{3}}{k} I_{0^{+}}^{q} D\left(f(t, u), 0_{E^{1}}\right), \\
& { }^{c} D_{0^{+}}^{1-q}(V(t, u(t))) \leq \frac{-c_{3}}{k} D\left(I_{0^{+}}^{q} f(t, u), 0_{E^{1}}\right), \\
& { }^{c} D_{0^{+}}^{1-q}(V(t, u(t))) \leq \frac{-c_{3}}{k} D\left(u(t), 0_{E^{1}}\right) .
\end{aligned}
$$
get

Finally, by applying $c_{1}, c_{2}$, and $\left(-c_{3} / k\right)$ in Theorem 1 , we

$$
D\left(u(t), 0_{E^{1}}\right) \leq \frac{V(0)}{c_{1}} E_{1-q}\left(-\frac{c_{3}}{c_{2} k} t^{1-q}\right),
$$

where $V(0)=V(0, u(0))$.

Finally, all the conditions of Theorem 1 are satisfied; thus, it follows that problem (47) is Mittag-Leffler stable.

\section{Conclusion and Future Works}

In this paper, we studied the stability result of fuzzy fractional differential equations by introducing Mittag-Leffler stability notion. Indeed, we discussed some sufficient criteria to demonstrate the stability of the trivial solution of the proposed system.

Our future work is to establish the Mittag-Leffler stability of intuitionistic fuzzy multivariable nonlinear fractional systems.

\section{Data Availability}

The data used to support the findings of this study are available from the corresponding author upon request.

\section{Conflicts of Interest}

The authors declare that they have no conflicts of interest.

\section{References}

[1] R. P. Agarwal, V. Lakshmikantham, and J. J. Nieto, "On the concept of solution for fractional differential equations with uncertainty," Nonlinear Analysis, vol. 54, no. 72, pp. 2859-2862, 2010.

[2] S. Arshad and V. Lupulescu, "On the fractional differential equations with uncertainty," Nonlinear Analysis, vol. 74, pp. 3685-3693, 2011.

[3] V. Lupulescu, "Fractional calculus for interval-valued functions," Fuzzy Sets and Systems, vol. 25, no. 265, pp. 63-85, 2015.

[4] R. Alikhani and F. Bahrami, "Global solutions for nonlinear fuzzy fractional integral and integrodifferential equations," Communications in Nonlinear Science and Numerical Simulation, vol. 18, no. 8, pp. 2007-2017, 2013.
[5] T. Allahviranloo, A. Armand, and Z. Gouyandeh, "Fuzzy fractional differential equations under generalized fuzzy Caputo derivative," Journal of Intelligent and Fuzzy Systems, vol. 26, no. 3, pp. 1481-1490, 2014.

[6] T. A. Salahshour, S. Abbasbandy, and D. Baleanu, "Existence and uniqueness results for fractional differential equations with uncertainty," Advances in Difference Equations, vol. 112, pp. 1687-1847, 2012.

[7] S. Salahshour, T. Allahviranloo, and S. Abbasbandy, "Solving fuzzy fractional differential equations by fuzzy Laplace transforms," Communications in Nonlinear Science and Numerical Simulation, vol. 17, pp. 1372-1381, 2012.

[8] N. V. Hoa, V. Lupulescu, and D. O'Regan, "A note on initial value problems for fractional fuzzy differential equations," Fuzzy Sets and Systems, vol. 347, pp. 54-69, 2018.

[9] L.-L. Huang, D. Baleanu, Z.-W. Mo, and G.-C. Wu, "Fractional discrete-time diffusion equation with uncertainty: applications of fuzzy discrete fractional calculus," Physica A: Statistical Mechanics and Its Applications, vol. 508, no. 8, pp. 166-175, 2018.

[10] L. L. Huang, B. Q. Liu, D. Baleanu, and G. C. Wu, "Numerical solutions of interval-valued fractional nonlinear differential equations," European Physical Journal Plus, vol. 134, 2019.

[11] D. Matignon, "Stability results for fractional differential equations with applications to control processing in:IMACSSMC," 1996.

[12] R. Agarwal, D. O’Regan, and S. Hristova, "Stability of Caputo fractional differential equations by Lyapunov functions," Applications of Mathematics, vol. 60, no. 6, pp. 653-676, 2015.

[13] T. A. Burton and B. Zhang, "Fractional equations and generalizations of Schaefer's and Krasnoselskii's fixed point theorems," Nonlinear Anal, vol. 75, pp. 6485-6495, 2012.

[14] S. Momani and S. Hadid, "Lyapunov stability solutions of fractional integrodifferential equations," International Journal of Mathematics and Mathematical Sciences, vol. 2004, no. 47, pp. 2503-2507, 2004.

[15] L. A. Zadeh, "Fuzzy sets," Information and Control, vol. 8, no. 3, pp. 338-353, 1965.

[16] H. T. Nguyen, "A note on the extension principle for fuzzy sets," Journal of Mathematical Analysis and Applications, vol. 64, no. 2, pp. 369-380, 1978.

[17] O. Kaleva, "Fuzzy differential equations," Fuzzy Sets and Systems, vol. 24, pp. 301-317, 1987.

[18] D. Dubois and H. Prade, Fuzzy Sets and Systems: Theory and Applications, Academic Press, New York, NY, USA, 1980.

[19] M. L. Puri and D. A. Ralescu, "Differentials of fuzzy functions," Journal of Mathematical Analysis and Applications, vol. 91, no. 2, pp. 552-558, 1983.

[20] A. Kilbas, H. M. Srivastava, and J. J. Trujillo, "Theory and applications of fractional differential Equations," in NorthHolland Mathematical Studies 204, Ed van Mill, Amsterdam, Netheralnd, 2006.

[21] I. Podlubny, Fractional Differential Equations, Academic Press, New York, NY, USA, 1999. 